\title{
The Success Rate of Classic Laryngeal Mask Airway Insertion During General Anesthesia in Different Age Groups
}

\author{
Mirmohammadtaghi Mortazavi' ${ }^{1}$, Atefeh Shadi ${ }^{1}$, Masoud Parish ${ }^{1 *}$
}

\begin{abstract}
Objectives: Uncertainty about the effect of age on the success rate of laryngeal mask airway (LMA) insertion led us to conduct the present study to evaluate the success rate of LMA in airway management in different age groups.

Materials and Methods: A total of 183 patients, who were admitted to Shohada hospital (Tabriz, Iran) during 2018-2019, were enrolled in this descriptive, cross-sectional, prospective study. The patients were selected through the convenience sampling method and divided into 4 age groups. After anesthesia, patients were compared in terms of duration and number of LMA insertion attempts and LMA insertion complications. The data were analyzed using SPSS20 through one-way ANOVA and the Chi-square test at $P<0.05$ ).

Results: There were significant statistical differences between different age groups regarding resistance against LMA insertion $(P=0.008)$ so that insertion was most comfortable and easy in the first attempt in the adult group compared to other groups. However, no significant statistical difference was observed between different groups in terms of the rate of air leakage $(P=0.129)$, the duration of successful LMA insertion $(P=0.119)$, and the number of LMA insertion attempts $(P=0.091)$. Regarding LMA insertion complications, laryngospasm was significantly higher in the middle-age and aged group $(P=0.009)$, and blood observation on LMA was significantly higher in the pre-school and child group $(P=0.005)$.

Conclusions: According to the results of this study, no difference was found between different groups in terms of successful LMA insertion and post LMA insertion complications.

Keywords: Oxygenation, Ventilation, LMA, Complications
\end{abstract}

\section{Introduction}

Laryngeal mask airway (LMA) is a useful device that is designed for managing airways during general anesthesia and has become a common alternative to airway management. The device is easily used by inexperienced people and provides proper management of airways in patients with mechanical ventilation and those with spontaneous breathing. LMA can be highly helpful for the users due to the problems encountered in successful airway management, especially when it is performed by the novice users such as assistants and health care providers working in the trauma and emergency units (1-3).

There are many advantages to using an LMA over an endotracheal tube, the most important of which are the prevention of laryngospasm and non-irritation of the larynx. On the one hand, LMA insertion may be unsuccessful since it is blindly inserted without direct vision $(4,5)$. Difficult insertion of LMA in some people is another limitation of this device. Therefore, its proper insertion can ensure the maximum efficiency of using LMA in auxiliary ventilation. Previous research has shown that LMA is not properly inserted in one-third of attempts, and incorrect insertion can endanger the patients' life
$(6,7)$. Therefore, it is necessary to identify the factors affecting the incorrect insertion of this valuable device. In the meantime, obesity, head anatomy, neck problems, and airway trauma are among the factors influencing the improper insertion of LMA. However, the effects of other factors have not been precisely determined yet $(8,9)$.

The success rate of LMA insertion in different age groups has not so far been studied coherently. Laryngeal masks are mostly used in adults and there is no information about the success rate in different age groups. Given the importance of this subject, the present study aimed to evaluate the success rate of LMA insertion in airway management in four age groups. It is noteworthy that achieving acceptable results regarding its efficiency, ease of use, and complications can help determine in which age group LMA could be used more or with caution so that to reduce airway complications and problems.

\section{Materials and Methods \\ Study Design}

This descriptive, cross-sectional, prospective study was conducted between June 20, 2018 and November 20, 2019 at Shohada hospital affiliated with Tabriz University of Medical Sciences. The sample size was determined 
Key Messages

What Is the Current Knowledge?

- LMA has the least complications for ventilation and oxygenation.

- LMA can be inserted in a short time and help with timely oxygenation.

What Is the Innovation of This Study?

- LMA can be inserted in a shorter time in adults.

- LMA insertion is associated with complications in children and aged groups.

- LMA is easier and more comfortable for adults than other age groups.

based on the results of a pilot study on 5-patient groups. Successful LMA insertion was the primary outcome of this study. Considering $\alpha=0.05$, the power of $80 \%$, and an acceptable clinical difference, the final sample size was calculated to be 183 patients who were divided into 4 groups (10). The samples were enrolled in the study through the convenience sampling method according to inclusion and exclusion criteria. The inclusion criteria were an indication for LMA insertion and consent to participate in the study. On the other hand, the exclusion criteria included problems and pathologies of the pharynx, any anatomical problems of the mouth, throat, or larynx, pregnancy, hiatal hernia, patients prone to aspiration, and patients with airway problems. Further, other criteria were high airway resistance, patients with a body mass index of above 30, a history of cardiopulmonary problems, trauma and head and face injuries, any head and neck abnormalities, and people with airway problems in terms of intubation.

\section{Procedure}

Patients' airways were examined by an anesthesiologist the night before the surgery to ensure if they meet the inclusion criteria. They were then classified by age based on a study (11) entitled "Age-specific search strategies for Medline." Next, the participants were assigned to four groups of pre-school and child (2-12 years old), adolescent (13-18 years old), adult (19-45 years old), and middle-age and aged (45-80 years old). After entering the operating room, receiving $500 \mathrm{~mL}$ of normal saline intravenously, and undergoing hemodynamic monitoring (i.e., heart rate, systolic and diastolic blood pressure, and arterial oxygen saturation measurement), the patients were routinely anesthetized using the classic LMA (TUOREN, Henan Tuoren Medical Device Company). Anesthesia was performed and managed by two anesthesiologists with work experience of more than 5 years.

\section{Data Collection Tools}

The data collection tool had three parts:

1. Demographic information which included age and gender.
2. The tool determining the number of LMA insertion attempts (which is classified as easy insertion in the first attempt without any resistance, an easy insertion in the first attempt but with little resistance, slightly difficult insertion but successful in the second attempt), the duration and the number of attempts for successful LMA insertion, and the success rate of LMA insertion.

3. Complications associated with LMA insertion, including air leakage and other complications (e.g., abdominal distension examined through hearing and stomach percussion, laryngospasm, and blood observation on LMA).

\section{Statistical Analysis}

Finally, the collected data were analyzed by SPSS 20 in four groups using the one-way ANOVA test and presented as mean ( \pm standard deviation) and frequency (percentage) for quantitative and qualitative variables, respectively. Descriptive measures such as skewness and kurtosis indicators, as well as fitness and reasonableness of the standard deviation (compared to the mean) were evaluated as well, and $P$ values of less than 0.05 were considered significant in this study.

\section{Results}

The patients consisted of 130 males (71\%) and 53 females (29\%) in the age range of 26 months to 78 years with a mean age of $27.03 \pm 18.46$ years, a median of 26 years, and an interquartile range of 24 years. They were assigned to four age groups of pre-school and child (2-12 years old, $\mathrm{n}=44$ ), adolescent (13-18 years old, $\mathrm{n}=35)$, adult (19-45 years old, $n=69$ ), and middle-age and aged (45-80 years, $\mathrm{n}=35$ ). According to demographic characteristics, there was a statistically significant difference between different age groups in terms of demographic characteristics (Table 1).

The highest rate of slightly difficult but successful LMA insertion in the second attempt was observed in the preschool and child group (11.4\%), followed by the middleaged and aged group (5.7\%). The highest rate of easy LMA insertion in the first attempt but with little resistance was found in the middle-aged and aged group (14.3\%), followed by the pre-school and child group (11.4\%). In addition, the highest rate of easy LMA insertion in the first attempt without resistance was detected in the adolescent group (97.1\%) and then in the adult group (91.3\%). On the other hand, the lowest rate of easy LMA insertion in the first attempt without resistance was observed in the pre-school and child group (77.3\%). This rate was $80 \%$ in the middle-aged and aged group (Figure 1). Finally, the highest frequency of easy LMA insertion in the first attempt was found in the adult group which should be taken into account. However, slightly difficult LMA insertion in the second attempt was also detected in the pre-school and child group. According to ANOVA results, 
Table 1. Comparison of Demographic Characteristics of Participants

\begin{tabular}{|c|c|c|c|c|c|}
\hline \multirow{2}{*}{ Variable } & \multicolumn{4}{|c|}{ Groups $(\mathrm{N}=183)$} & \multirow{2}{*}{$P$ Value $^{a}$} \\
\hline & Preschool and Child $(n=44)$ & Adolescent $(n=35)$ & Adult $(n=69)$ & Middle Age and Aged $(n=35)$ & \\
\hline Age $(\mathrm{M} \pm \mathrm{SD})$ & $07.12 \pm 01.45$ & $15.43 \pm 02.20$ & $21.20 \pm 01.55$ & $49.44 \pm 01.29$ & 0.211 \\
\hline Gender: Male (N, \%) & $35-26.92 \%$ & $28-21.53 \%$ & $41-59.42 \%$ & $26-20 \%$ & 0.259 \\
\hline
\end{tabular}

Note. SD: Standard deviation; ANOVA: Analysis of variance.

a Applied test: ANOVA.

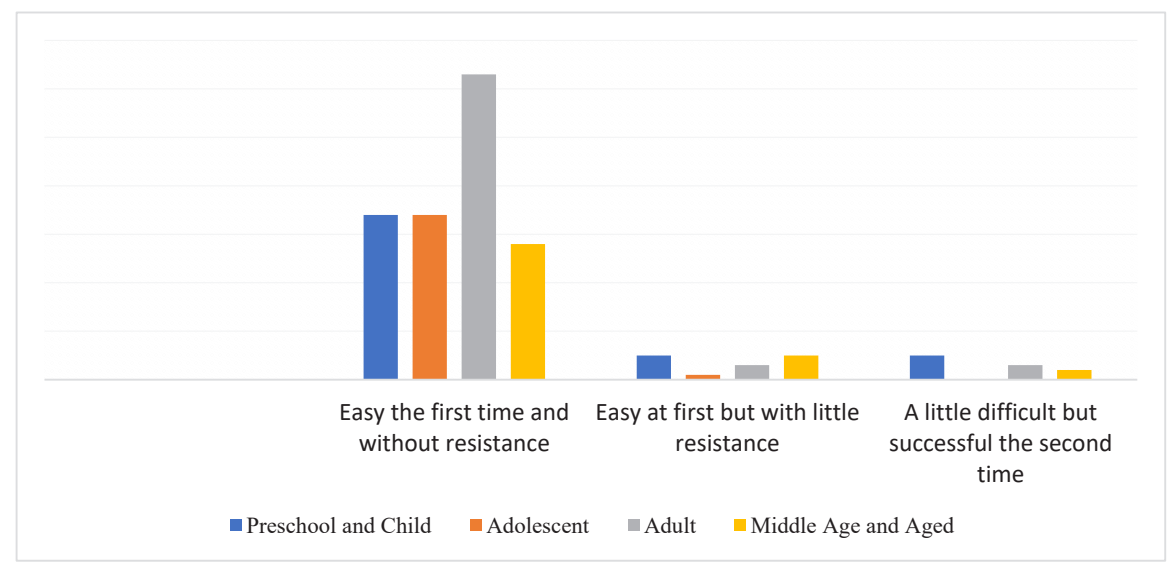

Figure 1. Resistance to LMA-embedding in Different Age Groups. Note. LMA: Laryngeal mask airway.

a significant statistical difference existed between different age groups in terms of the resistance to LMA insertion $(P=0.008)$ so that comfortable and easy LMA insertion in the first attempt was observed in the adult group.

Regarding the complications, there was no air leakage in 125 patients $(68.3 \%)$ while it was slight and intermediate in $50(27.3 \%)$ and $7(3.8 \%)$ patients and high in one patient $(0.5 \%)$. Regarding the duration of successful LMA insertion, the LMA was successfully inserted in $<15$ seconds in 163 patients (89\%) and $>15$ seconds in 19 patients (10.8\%) so that the LMA was successfully inserted in 5 seconds in 101 patients $(55.2 \%)$ and $>20$ seconds in only $4.2 \%$ of patients. The mask was successfully inserted in the first attempt in nearly 95\% of patients and after three attempts in only one patient $(0.55 \%)$, the related data of which are provided in Table 2. No statistically significant differences were found between the groups in terms of the air leakage rate $(P=0.129)$, the duration of successful insertion $(P=0.119)$, and the number of insertion attempts $(P=0.091)$.

Regarding LMA insertion complications, laryngospasm was observed in only one patient $(0.5 \%)$ and blood on LMA was found in two patients (1.1\%). Based on the results, no distention was found in the remaining 180 participants (98.4\%). The comparison of complications through ANOVA indicated that there were no statistically significant differences between different age groups (Table 3). The study of LMA-associated complications revealed statistically significant differences between different groups in terms of laryngospasm $(P=0.009)$ and blood observations on LMA $(P=0.005)$ while no statistically significant differences were detected between different age groups with regard to LMA-associated distension $(P>0.999)$.

\section{Discussion}

The present study aimed to examine the success rate of classic LMA insertion during general anesthesia in different age groups. The results demonstrated statistically significant differences between different age groups in terms of resistance to LMA insertion so that comfortable and easy insertion in the first attempt was mostly observed in the adult group compared with other groups. Therefore, LMA insertion in adults is extremely easier in comparison with other groups. The use of LMA has been considered as a convenient way to maintain mechanical ventilation in recent years and many anesthesiologists use this method for mechanical ventilation, especially in adults because it has been more successful in this group, which is in line with the results of our study. However, given that LMA can be used at different ages, its effects and success rate at different ages have not been studied yet, and anesthesiologists are cautious about using this method.

Similar studies investigated the success rate of LMA insertion and supraglottic gel device (I-GEL) on 61 ASA Class I-II patients aged 18-70 years who were admitted for minor orthopedic surgery with a surgery duration of less than 1 hour $(12,13)$. In this study, the success rate of LMA insertion was reported to be $80.6 \%$ and $12.9 \%$ in the first and second attempts, respectively. The rate of unsuccessful LMA insertion was $6.5 \%$. Our findings corroborate the 
Table 2. Comparison of the LMA Insertion Duration, the Number of Required Attempts, and the Leakage Rate Between Different Age Groups

\begin{tabular}{|c|c|c|c|c|c|c|}
\hline \multirow{2}{*}{ Variable } & & \multicolumn{4}{|c|}{ Groups $(\mathrm{N}=183)$} & \multirow[b]{2}{*}{$P$ Value $^{\mathrm{a}}$} \\
\hline & & $\begin{array}{c}\text { Preschool and Child } \\
n=44(\%)\end{array}$ & $\begin{array}{c}\text { Adolescent } \mathrm{n}=35 \\
(\%)\end{array}$ & Adult $n=69(\%)$ & $\begin{array}{c}\text { Middle Age and Aged } \\
n=35(\%)\end{array}$ & \\
\hline \multirow{4}{*}{ Leakage of air } & No leaks & $37-20.21$ & 18- 09.83 & 55- 30.05 & 20- 10.92 & \multirow{4}{*}{0.129} \\
\hline & Low leakage & $11-06.01$ & 9- 04.91 & 15- 08.19 & $15-08.19$ & \\
\hline & Medium leakage & $1-0.54$ & 2- 01.08 & 2- 01.08 & 2- 01.08 & \\
\hline & High leakage & $1-0.54$ & 0- 0 & 0- 0 & 0- 0 & \\
\hline \multirow{6}{*}{$\begin{array}{l}\text { Successful } \\
\text { embedding time }\end{array}$} & 5 seconds & $30-16.39$ & $15-08.19$ & 30- 16.39 & $12.01-10$ & \multirow{6}{*}{0.119} \\
\hline & 6 seconds & $1-0.54$ & $1-0.54$ & 2- 01.08 & $1-0.54$ & \\
\hline & 10 seconds & 6- 03.27 & 9- 04.91 & $25-13.66$ & 09.28- 10 & \\
\hline & 15 seconds & 0- 0 & $1-0.54$ & 2- 01.08 & 3- 01.62 & \\
\hline & 20 seconds & $0-0$ & 0- 0 & 2- 01.08 & 4- 02.16 & \\
\hline & More than 20 seconds & $1-0.54$ & $0-0$ & 3- 01.62 & 4- 02.16 & \\
\hline \multirow{3}{*}{$\begin{array}{l}\text { The number of } \\
\text { attempts }\end{array}$} & 1 time & $42-22.95$ & 33- 18.03 & $66-36.06$ & $32-17.48$ & \multirow{3}{*}{0.091} \\
\hline & 2 times & 2- 01.08 & 2- 01.08 & 3- 01.62 & $1-0.54$ & \\
\hline & 3 times & 0- 0 & $0-100$ & 0- 0 & $1-0.54$ & \\
\hline
\end{tabular}

Note. LMA: Laryngeal mask airway; ANOVA: Analysis of variance.

a Applied test: ANOVA.

Table 3. Comparison of LMA Insertion Complications Between Different Age Groups

\begin{tabular}{|c|c|c|c|c|c|}
\hline \multirow[b]{2}{*}{ Variable } & \multicolumn{4}{|c|}{ Groups $(\mathrm{N}=183)$} & \multirow[b]{2}{*}{$P$ Value $^{\mathrm{a}}$} \\
\hline & $\begin{array}{c}\text { Preschool and Child } \\
n=44(\%)\end{array}$ & $\begin{array}{c}\text { Adolescent } \\
\mathrm{n}=35(\%)\end{array}$ & $\begin{array}{c}\text { Adult } \\
\mathrm{n}=69(\%)\end{array}$ & $\begin{array}{c}\text { Middle Age and Aged } \\
n=35(\%)\end{array}$ & \\
\hline Laryngospasm & $0-0 \%$ & 0- $0 \%$ & $0-0 \%$ & $1-0.54 \%$ & 0.009 \\
\hline Blood on LMA & $2-01.08 \%$ & $0-0 \%$ & $0-0 \%$ & $0-0 \%$ & 0.005 \\
\hline Distension & $0-0 \%$ & $0-0 \%$ & $0-0 \%$ & $0-0 \%$ & $<0.999$ \\
\hline
\end{tabular}

Note. LMA: Laryngeal mask airway; ANOVA: Analysis of variance.

a Applied test: ANOVA.

theory that the overall success rate of LMA insertion is $100 \%$. The development of oral and dental status, the presence of teeth, and adequate moisture in the mouth in the adult group seem to be the reasons for the greater success of LMA insertion in this group compared to other age groups.

Regarding the complications associated with LMA insertion, the rate of the observed complications was extremely low. The only complication was laryngospasm, which was mostly observed in the middle-age and aged, as well as the pre-school and child groups (14), which is consistent with the results of the present study. The complications associated with MLA insertion were not common in similar studies. These complications are mostly observed in the aged and child groups compared to other groups (1), which matches the results of the present study. The use of the LMA seems to be a semi-invasive procedure and the LMA is soft, does not put pressure on the nerves in the tracheal area, and does not irritate the tracheal area. Finally, the method has the least side effects on the airway.

\section{Highlights}

The LMA insertion complications were higher in the children and aged groups compared to other age groups. It is highly easier to insert LMA in the adult group than in the other age groups.

Based on the results, there were no significant statistical differences between different age groups in terms of air leakage, the successful LMA insertion duration, and the successful attempt number. Other similar studies have also shown that LMA insertion is performed in a short time, and the rate of leakage after LMA insertion is often extremely low so that it poses no problem for ventilation and oxygenation. As regards the number of LMA insertion attempts, factors such as oral health, proper morphology of the mouth and teeth, and head and neck development are highly effective in this regard. The results of the present study are consistent with those of similar studies conducted in this field $(15,16)$. Considering that laryngeal mask placement does not require much skill and is easy to install, it would have been extremely easier for other skilled people if it had been embedded by skilled people. It was performed in a short time. It should also be noted that the amount of air leakage depends on the patient's weight, thus as the patient's weight increases, the size of the LMA should also be larger (the choice of size is based on weight) and if the size of the LMA is correctly chosen, the amount 
of leakage will naturally represent a decrease.

\section{Conclusions}

LMA can be used as a convenient and easy intubation method in all groups. The results of the present study indicated no difference between different groups in terms of successful LMA insertion and related complications. However, it is better to further apply this method in the adult group compared to other age groups.

\section{Limitations}

Failure to pay attention to the length of the operation, the lack of examination and attention to the presence/absence of artificial teeth, and the lack of mucosal examinations are some of the limitations of the present study.

\section{Suggestions for Future Studies}

Researchers in the present study suggest that after removing the limitations and weaknesses, this study be conducted on a larger sample size to determine its exact effectiveness in different age groups.

\section{Authors' Contribution}

MM: Study design, data analysis. AS: data collection. MP: article writing, data analysis, Reviewer response.

\section{Conflict of Interests}

Authors have no conflict of interests.

\section{Ethical Issues}

This study was performed according to the operating room routines. All steps (e.g., recording personal information and filling out the questionnaires) were performed with the informed consent of patients without inserting the patient's name (from the previous file) and all patient information was considered confidential throughout the study. This study was commenced after obtaining the code of ethics from the Ethics Committee of Tabriz University of Medical Sciences (IR.TBZMED.REC.1398.179) and the required permissions from the authorities of Shohada hospital, Tabriz University of Medical Sciences.

\section{Financial Support}

This study was granted by Tabriz University of Medical Sciences.

\section{Acknowledgments}

This study was extracted from a thesis by Dr. Atefeh Shadi (Medical Doctor) under the guidance of Dr. Mirmohammadtaghi Mortazavi (A faculty member). The researchers are grateful to the spiritual support provided by Tabriz University of Medical Sciences, as well as the patients who participated in the study.

\section{References}

1. de Carvalho ALR, Vital RB, de Lira CCS, et al. Laryngeal mask airway versus other airway devices for anesthesia in children with an upper respiratory tract infection: a systematic review and meta-analysis of respiratory complications. Anesth Analg. 2018;127(4):941-950. doi:10.1213/ane.0000000000003674
2. Driver BE, Martel M, Lai T, Marko TA, Reardon RF. Use of the intubating laryngeal mask airway in the emergency department: a tenyear retrospective review. Am J Emerg Med. 2020;38(7):1367-1372. doi:10.1016/j.ajem.2019.11.017

3. Xu R, Zhu Y, Fan Q, Shen X, Li WX. Comparison between the cobra perilaryngeal airway and laryngeal mask airways under general anesthesia: a systematic review and meta-analysis. Anesth Analg. 2017;125(3):958-966. doi:10.1213/ane.0000000000002316

4. van Esch BF, Stegeman I, Smit AL. Comparison of laryngeal mask airway vs tracheal intubation: a systematic review on airway complications. J Clin Anesth. 2017;36:142-150. doi:10.1016/j.jclinane.2016.10.004

5. Strametz R, Bergold MN, Weberschock T. Laryngeal mask airway versus endotracheal tube for percutaneous dilatational tracheostomy in critically ill adults. Cochrane Database Syst Rev. 2018;11(11):CD009901. doi:10.1002/14651858.CD009901.pub3

6. Song Z, Tan J, Fang J, Bian Q, Gu L. Comparison of laryngeal mask airway and endotracheal intubation in gynecological cancer operation. Oncol Lett. 2019;17(2):2344-2350. doi:10.3892/ol.2018.9813

7. Xu R, Lian Y, Li WX. Airway complications during and after general anesthesia: a comparison, systematic review and meta-analysis of using flexible laryngeal mask airways and endotracheal tubes. PLoS One. 2016;11(7):e0158137. doi:10.1371/journal.pone.0158137

8. Li L, Zhang Z, Yao Z, et al. The impact of laryngeal mask versus other airways on perioperative respiratory adverse events in children: a systematic review and meta-analysis of randomized controlled trials. Int J Surg. 2019;64:40-48. doi:10.1016/j.ijsu.2019.02.020

9. Chen WQ, Guo N, Wang SS, Wang R, Huang F, Li SR. General laryngeal mask airway anesthesia with lumbar plexus and sciatic block provides better outcomes than general anesthesia and endotracheal intubation in elderly patients undergoing hip surgery. Arch Gerontol Geriatr. 2018;78:227-232. doi:10.1016/j.archger.2018.07.005

10. Aghdashi MM, Valizade Hasanloei MA, Abbasivash R, Shokouhi S, Salehi Gharehvaran S. Comparison of the success rate of laryngeal mask air way insertion in classic \& rotatory methods in pediatric patients undergoing general anesthesia. Anesth Pain Med. 2017;7(2):e38899. doi:10.5812/aapm.38899

11. Eglen M, Kuvaki B, Günenç F, et al. [Comparison of three different insertion techniques with LMA-Unique ${ }^{\mathrm{TM}}$ in adults: results of a randomized trial]. Rev Bras Anestesiol. 2017;67(5):521-526. doi:10.1016/j.bjan.2017.04.007

12. Pournajafian A, Alimian M, Rokhtabnak F, Ghodraty M, Mojri M. Success rate of airway devices insertion: laryngeal mask airway versus supraglottic gel device. Anesth Pain Med. 2015;5(2):e22068. doi:10.5812/aapm.22068

13. Abedini $N$, Parish $M$, Farzin $H$, Pourfathi $H$, Akhsham $M$. The determination of an appropriate time for placement of the classic laryngeal mask airway in patients undergoing general anesthesia. Anesth Pain Med. 2018;8(2):e64427. doi:10.5812/aapm.64427

14. Mottaghi K, Eftekharian A, Salimi A, et al. Comparison of post intubation complications of endotracheal tube and laryngeal mask airway in pediatrics. Ann Anesth Crit Care. 2017;2(1):e10186. doi:10.5812/ aacc. 10186

15. Akhondzadeh R, Vojdani S, Aslani SM. Hemodynamic changes after intubation of endotracheal tube, LMA Classic ${ }^{\mathrm{TM}}$, and I-gel in patients candidates for elective eye surgery. Ann Anesth Crit Care. 2018;3(1):e11701. doi:10.5812/aacc.11701

16. Farhadloo R, Kashaninejad M, Hasanpour L, et al. A comparison of insertion and success rate in the use of two methods of endotracheal intubation and laryngeal mask airway. Qom Univ Med Sci J. 2016;10(10):34-40. [Persian].

Copyright $\odot 2022$ The Author(s); This is an open-access article distributed under the terms of the Creative Commons Attribution License (http://creativecommons.org/licenses/by/4.0), which permits unrestricted use, distribution, and reproduction in any medium, provided the original work is properly cited. 